\title{
ESPOROTRICOSE: RELATO DE CASO
}

\author{
Barbara Freitas Neves I \\ Laryssa Bezerra da Nóbregal \\ Marcela Vasconcelos Fernandes \\ Yohana Oliveira de Barros I \\ Luciana Cavalcante Trindade II
}

\begin{abstract}
RESUMO
A esporotricose, causada pelo fungo Sporothrix schenckii, é uma dermatomicose, subcutânea ou sistêmica, que acomete homens e animais, com destaque aos gatos. Com apresentação clínica diversa, pode acometer o homem sem predileção de sexo, faixa etária ou raça, com transmissão clássica, por meio da inoculação traumática do fungo na pele. O diagnóstico é feito através da história clínica e do isolamento do fungo no exame de cultura. O tratamento é realizado com solução de iodeto de potássio ou compostos azólicos e, geralmente, não há complicação. O presente artigo tem como objetivo relatar um caso clínico de esporotricose, ocorrido em João Pessoa/Paraíba, e realizar uma breve revisão de literatura a respeito dessa patologia e seus aspectos clínicos e laboratoriais. A paciente, com lesões sugestivas e história epidemiológica positiva, foi diagnosticada clinicamente com esporotricose na forma cutâneo-linfática e apresentou boa resposta terapêutica. Mesmo não sendo um agravo de notificação compulsória, a patologia trata-se de um problema de saúde pública, de modo que os profissionais de saúde devem estar atentos quanto à identificação das lesões e ao diagnóstico precoce.
\end{abstract}

\section{PALAVRAS-CHAVE}

Dermatomicose. Esporotricose. Itraconazol.

\section{INTRODUÇÃO}

A Esporotricose é uma micose subcutânea caracterizada por lesões nodulares que podem supurar ou ulcerar. Tem evolução subaguda ou crônica na maior parte dos casos. É causada pelo fungo Sporothrix schenckii que afeta principalmente homens e animais, em especial os gatos. É a mais frequente das micoses subcutâneas ${ }^{1}$.

O agente apresenta distribuição mundial e a doença é considerada endêmica na América Latina. É apontada como a micose sucbutânea mais comum na América do Sul. No que tange ao Brasil, apresenta-se de forma endêmica na região Sudeste do país².
A esporotricose pode acometer o ser humano de ambos os sexos, de qualquer faixa etária ou raça, independentemente de fatores individuais predisponentes, podendo gerar surtos familiares. O modo de transmissão clássico ocorre pela inoculação traumática do fungo na pele, sendo, por vezes, classificada como doença de cunho ocupacional, no que profissões - como floristas, agricultores, jardineiros, caçadores, veterinários - assumem maior risco de aquisição da infecção. Outras formas de transmissão são descritas, como inalação dos fungos e transmissão zoonótica, através da arranhadura ou mordedura de animais 
doentes, especialmente felinos ${ }^{2}$.

Como a transmissão por felinos tem sido relatada como a forma mais comum, veterinários, técnicos e donos de gatos são considerados como novas categorias de risco para adquirir a micose 3 .

O local de inoculação do fungo mais frequente são os membros superiores $(75,5 \%)$, seguido dos membros inferiores (10\%), da cabeça ( $9 \%$ ) e do $3,2 \%$ do tronco 4.

A apresentação clínica da doença é muito variada, observa-se desde nódulos isolados a lesões múltiplas disseminadas. A forma clínica apresentada, após a contaminação, depende de diversos fatores, como a carga do inóculo, a profundidade da inoculação traumática, a tolerância térmica da cepa e a resposta imunológica do hospedeiro. As lesões costumam ser restritas a pele, tecido celular subcutâneo e vasos linfáticos adjacentes. Em raras ocasiões, podem disseminar-se para outros órgãos, ou ainda ser primariamente sistêmica, resultante da inalação de esporos 5 .

O diagnóstico baseia-se na história clínica e no isolamento do fungo, através de cultura, nos meios de Ágar Sabouraud-dextrose ou Ágar-dextrose-batata².

Diversas modalidades de tratamento para esporotricose são descritas, desde o calor local ao uso da solução saturada de lodeto de Potássio e de antifúngicos como Itraconazol, com taxas de sucesso terapêutico variáveis na literatura médica ${ }^{2}$.

Embora não seja doença de notificação compulsória, estudos recentes ${ }^{6}$ e dados de institutos de pesquisa para o público leigo mostram que, nos últimos anos, tem havido aumento no número de casos de esporotricose entre animais domésticos em zonas urbanas, como no Rio de Janeiro7. A mesma situação parece estar ocorrendo em outras cidades, como João Pessoa/PB.

Considerando a esporotricose como uma micose zoonótica importante aos animais e homens, o estudo tem como objetivo relatar um caso de uma paciente diagnosticada com esporotricose, avaliando as características clínicas, a forma de transmissão, a evolução, o diagnóstico e o tratamento.

\section{RELATO DE CASO}

Paciente do sexo feminino, 33 anos, parda, natural de Guarabira e residente no bairro Funcionários - João Pessoa/PB, ensino médio completo, do lar, casada. Procurou a Unidade de Saúde Familiar (USF) de sua região em 21/07/2016, queixando-se de lesão ulcerada em segundo quirodáctilo da mão direita, tendo sido encaminhada da USF ao Complexo Hospitalar de Doenças Infectocontagiosas Clementino Fraga, em sua cidade.

Foi atendida no ambulatório de Dermatologia Sanitária do referido hospital no dia 29/07/2016, com relato de lesão em indicador direito, há um mês, dolorosa e pruriginosa, sem melhora, após uso de antibiótico (não sabia o nome). Associada- mente, passara a apresentar, na evolução, "caroços" em antebraço direito. Associava o surgimento da lesão à perfuração com pedaço de madeira do quintal de sua residência. De dados relevantes nos Antecedentes e Hábitos de Vida/Condições Sócio-econômico-culturais, referia habitar com mais três pessoas (marido e filhos), em uma casa de alvenaria e com água encanada, rua não pavimentada e sem rede de saneamento básico; possuía animais domésticos - cachorro e gato. O felino apresentara, no mesmo período, ferida em cavidade nasal. Negava ter sofrido arranhadura ou mordedura dos animais. Negava afecções pregressas e uso contínuo de medicação, etilismo e tabagismo. Refe- 
ria que o filho também desenvolvera uma lesão cutânea em região infraescapular esquerda.

Ao exame físico, a paciente apresentava uma placa eritemato-infiltrada, acometendo a falange média do indicador direito, encimada por uma úlcera de fundo raso e vegetante, e exsudação serosa em pequeno volume. Percebiam-se também nódulos indolores, de tamanho variado (1 a $2 \mathrm{~cm}$ de diâmetro), com pele suprajacente sã ou eritematosa, localizados no dorso da mão direita e face dorsal do antebraço direito (até o terço médio), em disposição linear, formando um cordão ascendente, no trajeto da drenagem linfática (figuras 1 e 2).

A partir dos dados de anamnese, com história epidemiológica positiva-exposição a felino apresentando ferimento e

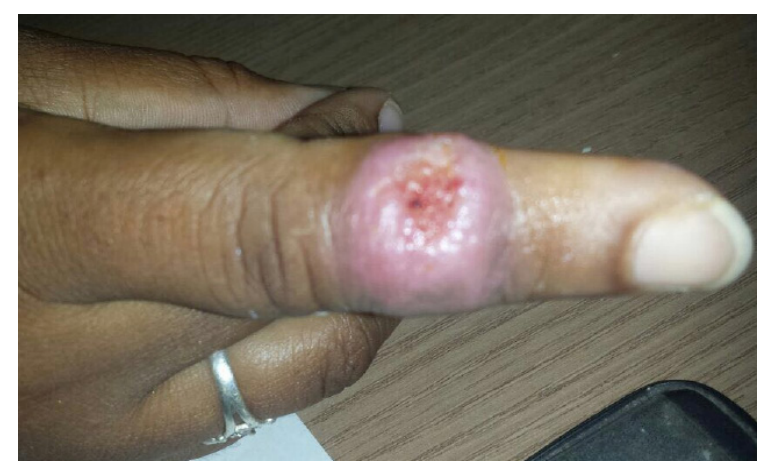

Figura 1 - Placa eritemato-infiltrada acometendo a falange média do indicador direito, encimada por uma úlcera de fundo raso e vegetante (primeira consulta).

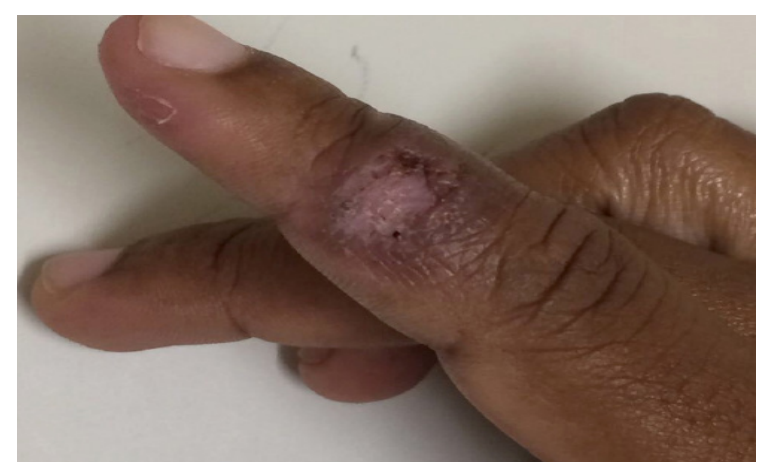

Figura 3 - Lesão em cicatrização (sessenta dias após tratamento). trauma com pedaço de madeira de quintal, associada ao exame físico, foi feito o diagnóstico clínico de esporotricose na forma cutâneo-linfática. Foi prescrito Itraconazol 10omg, 2 comprimidos ao dia e orientada a realizar avaliações mensais. Na mesma consulta, foi avaliado o filho da paciente, de 8 anos de idade, que também apresentava lesão sugestiva de esporotricose forma cutânea - sendo prescrito lodeto de Potássio solução.

Em reavaliação médica no dia 23/09/2016, mãe e filho mostravam-se com significativa melhora, tendo sido mantida a conduta (figura 3). No dia 24/11/2016, a paciente retornou com lesão totalmente cicatrizada e filho também apresentava cicatrização completa. A conduta tomada foi de mais sessenta dias de tratamento para ambos (figura 4).

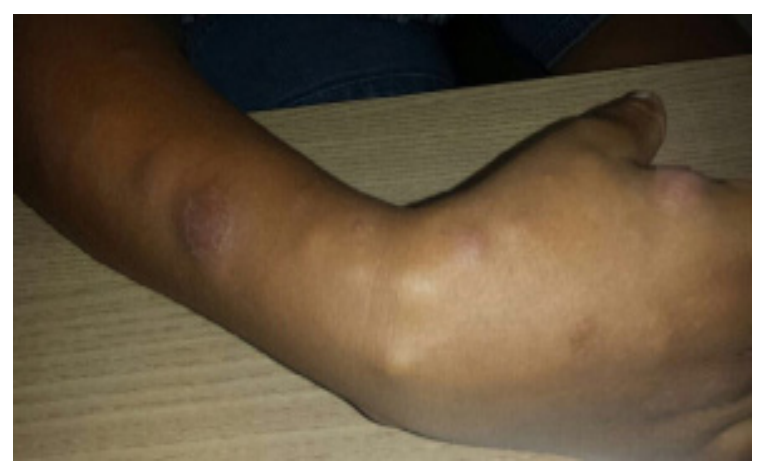

Figura 2 - Nódulos no dorso da mão direita e face dorsal do antebraço direito (até o terço médio), formando um cordão ascendente, no trajeto da drenagem linfática (primeira consulta).

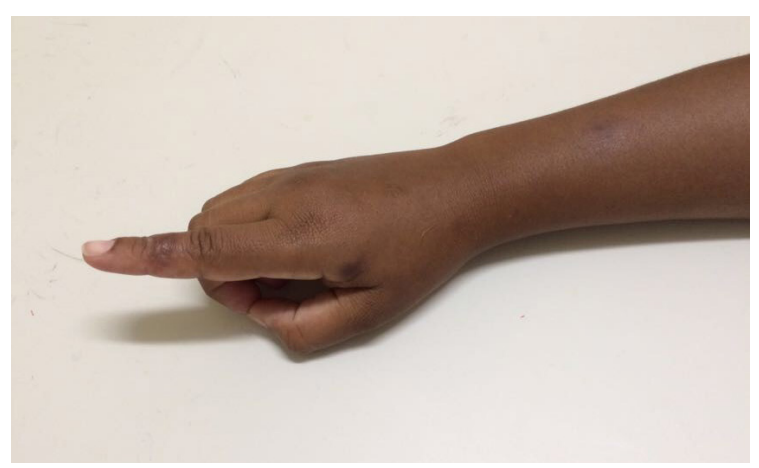

Figura 4 - Cicatrização quase completa (cento e vinte dias de tratamento). 
Em janeiro/2017, a paciente e o filho retornaram com lesões totalmente cicatrizadas e o tratamento foi suspenso (figura 5 ).

No dia 21 de março de 2017, voltaram para reavaliação, mantendo a excelente resposta terapêutica e a conduta foi de alta ambulatorial para ambos.

\section{DISCUSSÃO}

Foi apresentado um caso de esporotricose na forma cutânea-linfática, em uma paciente imunocompetente, ocorrido no município de João Pessoa, estado da Paraíba, com boa resposta à terapêutica estabelecida.

Estudos realizados em outros estados, mostraram o aumento no número de casos de esporotricose nas últimas décadas, considerada como a doença de transmissão zoonótica mais comum 9 . É um micose de apresentação cutânea, subcutânea ou sistêmica, podendo evoluir da forma subaguda à crônica. É causada pelo fungo dimórfico Sporothrix schenckii, que pode acometer o homem e espécies de animais variadas. As manifestações clínicas mais comuns são as apresentações cutânea localizada e cutânea-linfática 8,11 , que foram as ocorridas no caso em tela. Apesar de habitarem o mesmo domícilio, onde possivelmente ocorreu a transmissão do fungo, a paciente e o seu filho desenvolveram quadros clínicos diferentes, o que parece estar relacionado a fatores individuais do hospedeiro ${ }^{2}$.

A paciente em questão é uma mulher adulta, que relatava desenvolver, exclusivamente, atividades domiciliares. Embora a esporotricose possa acome-

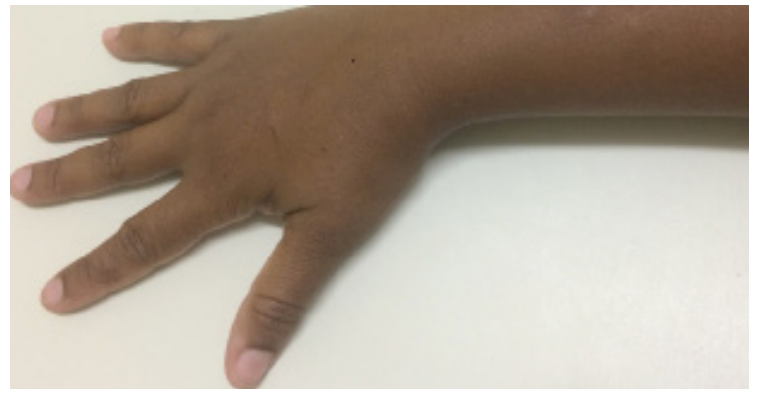

Figura 5 - Cicatrização completa, após 6 meses de tratamento.

ter indivíduos de ambos os sexos, de qualquer faixa etária ou raça, independentemente de fatores individuais predisponentes, em um estudo, desenvolvido por Silva e colaboradores, foi percebido o predomínio da doença em mulheres na faixa etária dos 21 aos 60 anos de idade e fora do ambiente do mercado de trabalho, o que os autores acreditam ser um indicativo da transmissão domiciliar9, como parece ter ocorrido no caso descrito.

É classificada como doença de cunho ocupacional, em que floristas, agricultores e jardineiros assumem o risco de aquisição da doença. Porém, estão mais propensos os profissionais que lidam com animais contaminados, como os veterinários ${ }^{2,10}$. No entanto, vêm sido descritas, nos últimos anos, epidemias em algumas cidades, como o Rio de Janeiro, relacionadas a felinos domésticos, inclusive com surtos famili$\operatorname{ares}^{9}$, à semelhança do que ocorreu no caso apresentado.

A respeito da transmissão em condições não ocupacionais, são importantes na epidemiologia, tanto o trauma após contato com plantas e areia, em casas com quintal ${ }^{2,5}$, quanto o contato direto com as lesões do felino enfer- 
mo, o que proporcione a contaminação, através de uma solução de continuidade cutânea humana preexistente ${ }^{10}$. No caso em questão, a paciente relatou trauma no seu quintal e contato com felino apresentando dermatose; é possível então que a inoculação tenha ocorrido com o pedaço de madeira do quintal, ou através do seu animal doméstico infectado, pela solução de continuidade já existente.

O diagnóstico baseia-se na anamnese e no exame clínico, essencialmente, e no isolamento do fungo, através de cultura, nos meios de Ágar Sabouraud-dextrose ou Ágar-dextrose-batata, incubados e observando-se o crescimento das colônias por 30 dias. Pode-se lançar mão do exame histopatológico, não específico, que evidencia hiperplasia pseudoepiteliomatosa e reação granulomatosa, que pode conter microabscessos no seu interior. Colorações especiais podem propiciar o achado de formas leveduriformes do agente, com estruturas características e esporos em brotamento. Já os exames sorológicos mostram-se úteis para as formas atípicas e extracutâneas ${ }^{2}$. No caso apresentado, o diagnóstico ocorreu após a história clínica e exame físico bem apurados, sendo constatada a forma típica cutâneo-linfática e a presença de dados epidemiológicos positivos. Não foi realizado estudo complementar - micológico ou sorológico - pela indisponibilidade na rede de saúde. A esse respeito, Barros e colaboradores relatam que, no quadro clínico característico da forma cutâneo-linfática e com história epidemiológica, é possível o diagnóstico, devendo ser encaminhados a unidades de referência somente casos de maior complexidade e formas atípicas 5 .

O tratamento é realizado com o antifúngico Itraconazol, na dose de 100 a 200mg ao dia2,9, considerado o fármaco de eleição, porém com o mesmo grau de recomendação que o lodeto de Potássio ${ }^{2}$. No entanto, embora se tenha demonstrado eficaz no tratamento humano e em animais, principalmente felinos, há alguns relatos de casos isolados resistentes ao Itraconazol, resultando em falha terapêutica ${ }^{8}$. Outra opção é a terbinafina, uma alilamina, que vem sendo avaliada quanto a sua atividade em relação ao Sporothrix schenckii e demonstrando intensa ação in vitro, frente ao agente. Atualmente também vem sendo estudada para o tratamento da esporotricose em humanos ${ }^{10}$. O tempo de uso do antimicótico está relacionado a resposta clínica, que ocorre, em média, em 12 semanas 5 , sendo indicada a manutenção do tratamento por 30 a 60 dias, após a cicatrização completa das lesões dermatológicas. O tratamento da paciente em questão foi conduzido, conforme a literatura vigente, e resultou em uma boa resposta à terapêutica.

Embora a esporotricose não seja um agravo de notificação compulsória, o que dificulta estabelecer a sua incidência real ${ }^{2}$, trata-se de problema de saúde pública5, cuja ocorrência aumentou em estados brasileiros nos últimos anos, à semelhança do que vem sendo percebido por relatos de profissionais no município de João Pessoa. Assim, este trabalho visa alertar os profissionais de saúde, os gestores e a população quanto às características da doença e o vínculo epidemiológico com gatos, de modo a estimular a identificação de lesões e o diagnóstico precoce da dermatose, bem como o tratamento dos indivíduos acometidos e dos felinos infectados. 


\title{
SPOROTRICHOSIS: CASE REPORT
}

\begin{abstract}
Sporotrichosis, caused by the fungus Sporothrix schenckii, is a dermatomycosis, subcutaneous or systemic, that affects human and animals, with emphasis on cats. With diverse clinical presentation, it can affect the human without predilection of sex, age group or race, with classical transmission through the traumatic inoculation of the fungus on the skin. The diagnosis is made through the clinical history and the isolation of the fungus through analysis. Treatment is performed with potassium iodide solution or azole drugs and usually there is no complication. The present article has the point to report a clinical case of sporotrichosis occurred in João Pessoa / Paraíba and to carry out a brief review of the literature on this pathology and its clinical and laboratory aspects. The patient, with suggestive lesions and a positive epidemiological history, was diagnosed clinically with sporotrichosis in the cutaneous-lymphatic form and presented a good therapeutic response. Even though it is not a compulsory notification disease, the pathology is a public health problem, so health professionals should be aware of the identification of the lesions and the early diagnosis.
\end{abstract}

\section{KEYWORDS}

Dermatomycosis. Sporotrichosis. Itraconazole.

\section{REFERÊNCIAS}

1 Muniz AS, Passos JP. Esporotricose Humana: conhecendo e cuidando em enfermagem. Revista de Enfermagem UERJ. abri./ jun. 2009. [Acesso em: 20 de novembro de 2017]. 17(2): [268-72]. Disponível em: <http:// www.facenf.uerj.br/v17n2/v17n2a23.pdf >.

2 Cordeiro, FN. et al. Ocorrência familiar de esporotricose zoonótica. Anais Brasileiro de Dermatologia, 2011. Acesso em: 20 de novembro de 2017. 86(S1): [121-24]. Disponível em: < http://www.scielo.br/pdf/abd/ v86n4s1/v86n4s1a32.pdf >.

3 Hugo CP, Rocha RDR, Ferreira MFR. Esporotricose Humana: aspectos clínicos, laboratoriais e caso clínico. Pós em Revista. 2013. [Acesso em: 21 de novembro de 2017]. 6(27): [217-22]. Disponível em: <http://docplayer. com.br/19382787-Esporotricose-humana-aspectos-clinicos-laboratoriais-e-caso-clinico. html>.

4 Esteves JA. Cabrita JD. Nobre GN. Micologia Médica. $2^{\text {a }}$ ed. Lisboa: Fundação Caloute Gulbenkian; 1990.
5 Barros MBL. et al. Esporotricose: a evolução e os desafios de uma epidemia. Revista Panamericana de Salud Pública. 2010. [Acesso em: 20 de novembro de 2017]. 27(6): [455-60]. Disponível em: <http://www.scielosp.org/pdf/rpsp/ v27n6/07.pdf>.

6 Reis BD. et al. Esporotricose em localização incomum: relato de um caso. Anais Brasileiro de Dermatologia, 2015. [Acesso em: 20 de novembro de 2017]. 90(3S1): [836]. Disponível em: <http://www.anaisdedermatologia.org.br/detalhe-artigo/102318/ Esporotricose-em-localizacao-incomum--relato-de-um-caso->.

7 Brasil, Fundação Oswaldo Cruz. Esporotricose: pesquisadores esclarecem sobre a doença, que pode afetar animais e humanos. Notícias, 25/06/2015. Acesso em: 20 de novembro de 2017. Disponível em: <https://portal.fiocruz.br/pt-br/content/ esporotricose-pesquisadores- esclarecem-sobre- doenca-que-pode-afetar- animais-e- humanos\&gt $>$. 
8 Meinerz ARM. et al. Suscetibilidade in vitro de isolados de Sporothrix schenckii frente à terbinafina e itraconazol. Rev. Soc. Bras. Med. Trop, 2007. [Acesso em: 21 de novembro de 2017]. 40(1): [60-2]. Disponível em: <http://www.scielo.br/pdf/rsbmt/v40n1/a12v40n1.pdf>.

9 Silva MBT. et al. Esporotricose urbana: epidemia negligenciada no Rio de Janeiro, Brasil. Cadernos de Saúde Pública, 2012. [Acesso em: 21 de novembro de 2017]. 28(10): [186780]. Disponível em: < http://www.scielo.br/ $\mathrm{pdf} / \mathrm{csp} / \mathrm{v} 28 \mathrm{n} 10 / 06 . \mathrm{pdf}>$.
10 Antunes, TA. et al. Esporotricose cutânea experimental: avaliação in vivo do itraconazol e terbinafina. Rev. Soc. Bras. Med. Trop, 2009. [Acesso em: 21 de novembro de 2017]. 42(6):[706-10]. Disponível em: < http://www. scielo.br/pdf/rsbmt/v42n6/18.pdf >.

11 Larsson CE. Esporotricose. Braz. J. Vet. Res. Anim. Sci, São Paulo, 2011. [Acesso em: 20 de novembro de 2017]. 48(3): [250-59]. Diponível em: < http://www. journals.usp.br/bjvras/article/download/34389/37127>. 